\title{
A CHECKLIST OF INCUNABULA IN THE \\ DEPARTMENT OF SPECIAL COLLECTIONS \\ AND ARCHIVES, A. S. ALEXANDER LIBRARY, RUTGERS UNIVERSITY
}

\section{BY THOMAS KNOLES}

Dr. Knoles is a former member of the Department of Special Collections and Archives. $\mathrm{He}$ is currently teaching English in Rutgers College.

This first listing of the incunabula at Rutgers is in a sense representative of its rare book collection as a whole. Both are in large part the result of the attentions of generous donors who have given books and/or the money with which to buy them. A few comments about the system employed may be helpful.

I. Authors, titles and printers are given according to the forms used by Frederick R. Goff in his Incunabula in American Libraries, rev. ed. (Millwood, N.Y.: Kraus Reprint Co., I973), hereafter referred to as "Goff"; Goff's forms are based on the standard modes of reference used in the major bibliographies of incunabula. Where they exist, references are also given to the unfinished but more thorough Gesamtkatalog der Wiegendrucke (Leipzig, etc., I925+), hereafter referred to as "GW."

2. The Goff number given with each entry will refer the reader to other relevant publications concerning the item, and the entry in Goff is therefore the simplest starting point for those seeking additional bibliographical information.

3. An asterisk $\left(^{*}\right)$ after a Goff number means that the Rutgers copy is not reported in Goff. It is hoped that this list will have the added benefit of supplementing Goff's listings of Rutgers holdings - fifteen of thirty-one titles here are not reported by Goff.

4. References are given here to discussions of the Rutgers copies of some works in the Journal of the Rutgers University Library. "G\&A" after such a citation means that the reference is to a brief notice of the acquisition of the work in the Journals "Gifts and Acquisitions" column (in existence beginning with v. I 2 [ I 948]) or its predecessor, "Notes from the Library." 
5. The following additional abbreviations are used:

ill. printed illustrations

p.d. printer's device

dec. hand-drawn decoration, including supplied or illuminated capitals, and/or paragraph marks

I. ALBER'TUS MAGNUS, I I 93?-I 280.

Mariale.

[Basel: Michael Wenssler, not after I 474.]

Fo.

$3 \mathrm{I} \mathrm{cm}$.

dec.

JRUL, 27 ( I 964), 52 (G\&A).

GW 679, Goff A-273 (one of 5 copies reported in the U.S.)

Clarence Garretson ('9I) Fund.

2. ANTONINUS FLORENTINUS, I 389-I 459.

De censuris.

[Mantua: Paulus de Butzbach, about I 475.]

$4^{\circ}$. $\quad 25 \mathrm{~cm}$. dec., ms notes.

JRUL, 3 I ( I 968), 66 (G\&A).

GW 2068, Goff A-775* (3 copies reported in the U.S.)

Julia B. Williamson Fund.

3. BALBUS, JOHANNES, d. I 298.

Catholicon.

Mainz: [Eponymous press (Johann Gutenberg?)] I 460.

$f^{\circ}$.

$37 \mathrm{~cm}$.

One leaf only; in Margaret Bingham Stillwell, Gutenberg and the Catholicon of 1460 (New York: E. B. Hackett, I 936).

$J R U L$, I ( I 937), 28 (G\&A).

GW 3 I 82 , Goff B-20*

Gift of Gabriel Wells.

4. BARTHOLOMAEUS DE CHAIMIS, d. ca. I 496.

Confessionale.

[Nuremberg: Fratres Eremitarum S. Augustini] 3 I May I480. $4^{\circ}$.

$22 \mathrm{~cm}$.

GW 6546, Goff B- I 59

Gift or Frank H. Stobaeus, I 880. 
5. CATO, DIONYSIUS.

Disticha de moribus, etc.

(with commentary by Robertus de Euremodio)

Antwerp: Gerard Leeu, 30 Oct. I 487.

$4^{\circ}$. $20 \mathrm{~cm}$. ill., dec., ms notes.

$J R U L, 24$ (I96I), I I4 (G\&A).

GW 6287, Goff C-299a (only copy reported in the U.S.)

Charles H. Brower ('25) and Mary Elizabeth Brower Fund.

6. CICERO, MARCUS TULLIUS, IO6-43 B.C.

Epistolae selectae.

[Rome: Bartholomaeus Guldinbeck, about I 485.]

$4^{\circ}$. $22 \mathrm{~cm}$. ms notes.

JRUL, 24 (I96I), I I4 (G\&A).

GW 687 I, Goff C-538* (as John F. Fleming copy, the only copy reported in the U.S.)

Gift of John F. Fleming.

7. CLAUDIANUS, CLAUDIUS.

Opera.

(edited by Thaddaeus Ugoletus)

Parma: Angelus Ugoletus, 23 Apr. I 493.

$4^{\circ}$. $20 \mathrm{~cm}$. p.d.

JRUL, 26 (1962), 3 I (G\&A).

GW 7060, Goff C-702*

Gift of James Duncan Pitney.

8. COLUMELLA, LUCIUS JUNIUS MODERATUS.

Hortuli commentarium, sive In de rustica lib. x commentum.

(anonymous commentary, without text)

[Rome: Bartholomaeus Guldinbeck, about I 485.]

$4^{\circ}$. $22 \mathrm{~cm}$. ms notes.

GW 7I 88, Goff C-765 (only copy reported in the U.S.)

Source unknown.

9. CRESCENTIIS, PETRUS DE, I230?-I320?

Ruralia commoda.

Louvain: Johann de Paderborn (Westphalia), 9 Dec. I474.

$\mathrm{f}^{\circ}$. $28 \mathrm{~cm}$. dec.

GW 782 I, Goff C-966

Source unknown. 
Io. CRESCENTIIS, PETRUS DE, I 230?-I320?

Ruralia commoda.

[Speier: Peter Drach, about I 490-95.]

f. $\quad 30 \mathrm{~cm}$. ill., ms notes.

JRUL, 6 ( I 943), 33-44.

GW 7825, Goff C-969

Funds of George H. Cook and Edward B. Voorhees (Class of I 88 I).

I I. DANTE ALIGHIERI, I265-I 32 I.

La Commedia.

(in Italian; commentary by Christophorus Landins; edited by Piero Figino. Also includes $I l$ Credo)

Venice: Petrus de Quarengiis, Bergomensis, I I Oct. I 497. $f^{\circ}$. $30 \mathrm{~cm}$. ill.

GW 7972, Goff D-35.

Gift of E. Byrne Hackett.

I 2. FLISCUS, STEPHANUS, fl. I 450.

Sententiarum variationes, seu Synonyma.

(in Latin and French; also includes Gasparinus Barzizius, De eloquentia; pseudo-Cicero, Synonyma)

[Paris: Philippe Pigouchet, after I 49 I.]

$4^{\circ}$ 20cm. dec., ms notes.

JRUL, 30 ( 1967), 60 (G\&A).

GW IOO I $5^{*}$ (not in Goff; GW reports 2 copies, neither in U.S.)

Clarence Garretson ('9I) Fund.

13. GERSON, JOHANNES, I 363-I 429

Opus tripartitum de praeceptis Decalogi, de confessione, et de arte moriendi.

[Cologne: Ulrich Zel, about I 467.]

$4^{\circ}$. $22 \mathrm{~cm}$. dec.

JRUL, 24 (1964), I I 4 (G\&A).

Goff G-238* (reported as John F. Fleming copy)

Gift of John F. Fleming.

I 4. HERMES TRISMEGISTUS.

De potestate et sapienta Dei.

(Latin translation by Marsilius Ficinus)

Venice: Maximus de Butricis, 29 July I 49 I. 
$4^{\circ}$. $\quad \mathrm{Igcm}$. ms notes.

Goff $\mathrm{H}-8 \mathrm{O}$

Robert H. Pruyn (Class of I 833) Fund.

I 5. HIGDEN, RANULPHUS, d. I 364 .

Polychronicon.

One leaf only; in George Parker Winship. William Caxton and the First English Press (New York: E. B. Hackett, I 938).

$\mathrm{f}^{\circ}$.

Goff $\mathrm{H}-\mathrm{I} 67^{*}$ $3 \mathrm{Icm}$.

Appropriation of the State of New Jersey for Reference Books and Periodicals.

I6. HOMERUS.

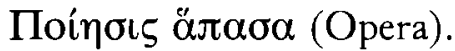

(in Greek; edited by Demetrius Chalconcylas)

Florence: Bernardus Nerlius, Nerius Nerlius and Demetrius Damilas, "9 Dec. 1488" [not before I3 Jan. I488/89]. $\mathrm{f}^{\circ}$. $3 \mathrm{Icm}$.

JRUL, 22 ( I958), 24 (G\&A).

Goff H-30O

Source unknown.

I 7. HORATIUS FLACCUS, QUINTUS, 65-8 B.C.

Opera.

(with commentary by Porphyrius and Acron)

Milan: Antonius Zarotus, for Augustinus Maria Conagus, I 485. $f^{\circ}$. $27 \mathrm{~cm}$. ms notes.

Goff H-452 (one of 3 copies reported in the U.S.)

Gift of Rev. John Forsyth (Class of I 829).

I 8. INNOCENTIUS VIII, PONT. MAX.

Bulla, 6 Jan. I $484 / 85$ (Sacrosanctum matrem ecclesiam).

[Vienna: Printer of the I 482 "Vocabolista" (Stephen Koblinger?), after 6 Jan. 1485 .]

$4^{\circ}$. $22 \mathrm{~cm}$.

Goff I-IO2* (reported as John F. Fleming copy)

Mr. and Mrs. Corlett H. Wilhelm Fund.

I 9. JACOBUS DE VORAGINE, fl. I 244- 298.

Legenda aurea sanctorum, sive Lombardica historia.

Lyons: Mathias Huss, 20 July 1487. 
$f^{\circ}$. JRUL, 24 (I96I), I I 4 (G\&A).

ill., p.d., dec., ms notes.

Goff J-I I 8* (as John F. Fleming copy, one of 4 copies reported in the U.S.)

Duncan Dunbar Sutphen (Class of 1 883) Fund.

20. JUSTINUS, M. JUNIANUS.

Epitomae in Trogi Pompeii historias.

Venice: Filippo di Pietro, I 2 Dec. I 479.

$\mathrm{f}^{\circ}$.

$28 \mathrm{~cm}$.

ms notes.

$J R U L$, Iо ( I 947), 33-34.

Goff J-6 I 8

Gift of the Gabriel Wells Estate.

21. MACER FLORIDUS.

Macer floridus de viribus herbarum carmen.

[Geneva: Unassigned, after I 500?]

$4^{\circ}$. 20cm. ill.

Goff $\mathrm{M}-4^{*}$ (one copy reported in the U.S.)

Funds of George H. Cook and Edward B. Voorhees (Class of I 88 I).

22. MAFFEUS, CELSUS, c. I 425- I 508. dinalium.

Monumentum compendiosum pro confessionibus Car-

Venice, Petrus de Quarengiis, Bergomensis, 22 Mar. I 498.

$4^{\circ}$.

$22 \mathrm{~cm}$.

JRUL, 30 ( I967), 60.

Goff (suppl.) M-I ga (only copy reported in the U.S.)

James W. Mailer Fund.

23. OVIDIUS NASO, PUBLIUS, 43 B.C-I 8 A.D.

De arte amandi et De remedio amoris.

(with commentary by Bartholomaeus Merula)

Venice: Joannes Tacuinus, de Tridino, 5 July I 494.

$\mathrm{f}^{\circ}$.

$3 \mathrm{Icm}$.

p.d.

JRUL, 24 ( I 96 I), I I 4 (G\&A).

Goff O-I 44 (one of 5 copies reported in the U.S.)

Gift of John F. Fleming.

24. PARKER, HENRY, d. I 470.

Dives and Pauper.

Westminster: Wynkyn de Worde, 3 Dec. I 496. 
f. $\quad 30 \mathrm{~cm}$. ill.; p.d.; ms notes.

JRUL, 30 ( г 967), 6 о (G\&A); 3 I ( т 967), г - го.

Goff p- I I $8 *$

Charles H. Brower ('25) and Mary Elizabeth Brower Fund. 25. PLATINA, BARTHOLOMAEUS, I 42 I-I 48 I .

De honesta voluptate et valetudine.

Cividale: Gerardus de Lisa, de Flandria, 24 Oct. I 480.

$4^{\circ}$. $23 \mathrm{~cm}$. ms notes.

Goff P-763* (reported as John F. Fleming copy)

Gift of John F. Fleming in honor of I. Robert Kriendler ('33). 26. REPERTORIUM DE PRAVITATE HAERETICORUM.

Valencia: [Lambert Palmart] i6 Sept. I 494.

$\mathrm{f}^{\mathrm{o}}$. $29 \mathrm{~cm}$.

$J R U L, 26$ ( I 962), 28-29; 3 I (G\&A).

Goff R-I 48

Charles H. Brower ('25) and Mary Elizabeth Brower Fund. 27. SCHEDEL, HARTMANN, I 440-I 5 I 4.

Liber chronicarum.

Augsberg: Johann Schoensperger, I Feb. I 497.

$f^{\circ}$.

Goff S-308

$3 \mathrm{Icm}$. ill., ms notes.

Gift of John C. Smock (Class of I 862).

28. SCRIPTORES HISTORIAE AGSTAE.

Venice: Joannes Rubeus Vercellensis, 15 July I 490.

$\mathrm{f}^{\circ}$.

$32 \mathrm{~cm}$.

ms notes.

Goff S- $342^{*}$

Source unknown.

29. SUETONIUS TRANQUILLUS, CAIUS, c. 70- I 22 ? A.D. Vitae XII Caesarum.

[Venice:] Nicolaus Jenson, I 47 I .

$4^{\circ}$. $29 \mathrm{~cm}$. dec.

JRUL, 22 ( I 958$), 25$ (G\&A).

Goff S-8 I 7

Duncan Dunbar Sutphen (Class of I 883) Fund. One of I 5 works printed by him in $147 \mathrm{I}$.

30. TURRECREMATA, JOHANNES DE, I388-I 468.

Tractatus de venerabili sacramento (de corpore Christi).

Delft: Jacob Jacobszoen van der Meer [about I 480]. 
$4^{\circ}$. $19 \mathrm{~cm}$.

JRUL, 28 ( I 964), 29 (G\&A).

p.d., dec., ms notes.

Goff T-557* (as John F. Fleming copy, one of 2 copies reported in the U.S.)

Charles H. Brower ('25) and Mary Elizabeth Brower Fund. 3 I. URBANUS BELLUNENSIS, c. I 443-I 524 .

Institutiones graecae grammaticae.

Venice: Aldus Manutius, Romanus, Jan. I $497 / 98$.

$4^{\circ}$. $22 \mathrm{~cm}$.

$J R U L, 24$ ( I 96 I), I I 4 (G\&A).

Goff U-66

Gift of Grace H. Talmadge.

\section{INDEX OF PRINTERS AND Publishers}

Butricis, Maximus, I 4

Butzbach, Paulus de, 2

Caxton, William, i 5

Conagus, Augustinus Maria,

\section{7}

Damilas, Demetrius, I6

Drach, Peter, IO

Fratres Erimitarum S.

Augustini, 4

Guldinbeck, Bartholomaeus, 6, 8

Gutenberg, Johann?, 3

Huss, Mathias, 19

Jenson, Nicolaus, 29

Koblinger, Stephen?, I 8

Leeu, Gerard, 5

Lisa, Gerardus de, de

Flandria, 25

Manutius, Aldus, Romanus, 3 I

Meer, Jacop Jacobszoen van der, 30

Nerlius, Bernardus, I 6
Nerlius, Nerius, I 6

Paderborn (Westphalia), Johann de, 9

Palmart, Lambert, 26

Pietro, Filippo di, 20

Pigouchet, Philippe, I 2

Printer of the "Catholicon," I 460 (Johann Gutenberg?), 3

Printer of "Vocabolista,"

(Stephen Koblinger?'), I 8

Quarengiis, Petrus de,

Bergomensis, I I , 22

Schoensperger, Johann, 27

Tacuinus, Joannes, de Tridino, 23

Ugoletus, Angelus, 7

Vercellensis, Joannes Rubeus, 28

Wenssler, Michael, I

Worde, Wynkyn de, 24

Zarotus, Antonius, I 7

Zel, Ulrich, I 3 


\section{Index by Place of Publication}

Austria

Vienna, I 8

Belgium

Antwerp, 5

Louvain, 9

England

Westminster, I 5, 24

France

Lyons, I 9

Paris, 12

Germany

Augsberg, 27

Cologne, I 3

Mainz, 3

Nuremberg, 4

Speier, Io
Holland

Delft, 30

Italy

Cividale, 25

Florence, I 6

Mantua, 2

Milan, I 7

Parma, 7

Rome, 6, 8

Venice I I, I4, 20, 22, 23,

$$
28,29,3 \mathrm{I}
$$

Spain

Valencia, 26

Switzerland

Basel, I

Geneva?, 2 I

\section{CORRECTION}

In the June I 983 issue of the Journal it was incorrectly stated that the photographic essay "Pacific Overtures" had originally appeared in Social Science and Modern Society. The correct name of the publication is Transaction/SOCIETY. We apologize to its editor for our oversight. 